\title{
Recent Development in Quantitative Electron Diffraction for Crystallography of Materials
}

\author{
Yoshitsugu Tomokiyo* and Syo Matsumura** \\ *Department of Materials Science and Engineering, Faculty of Engineering, \\ Kyushu University, Fukuoka 812-8581 Japan \\ **Department of Nuclear Engineering, Faculty of Engineering, \\ Kyushu University, Fukuoka 812-8581 Japan
}

\begin{abstract}
The present paper gives an extended review on applications of electron diffraction techniques to practical problems in materials science, such as (1) determination of structure factors and temperature factors by the critical voltage and intersecting Kikuchi line (IKL) methods, (2) measurement of local lattice parameters by convergent beam electron diffraction (CBED), (3) determination of partial degree of order in ternary alloys by IKL-ALCHEMI method, and (4) determination of structure factors by energy filtering CBED. The emphasis is placed on recent achievements of electron diffraction as a tool of quantitative crystallography of materials.
\end{abstract}

(Received March 6, 1998; In Final Form May 8, 1998)

Keywords: convergent beam electron diffraction, critical voltage effect, intersecting Kikuchi line method, atom location by channeling enhanced microanalysis, energy filtering, structure factor, lattice parameter, temperature factor, dynamical diffraction

\section{Introduction}

Atomic scattering factors for fast electrons are 3-4 orders of magnitude larger than those for X-rays, leading to sufficient intensity of electron diffraction even from a small area of thin specimen $^{(1)}$. Selected area electron diffraction and micro-beam electron diffraction are often employed complementarily to the bright- or dark-field imaging in transmission electron microscopy to identify the phase state of relevant local areas through analysis of crystallinity or crystal symmetry. If one would like to get quantitative information about the crystalline state, the well-established techniques in X-ray crystallography based on the means of kinematical diffraction is not straightforwardly applicable to electron diffraction, because the diffracted intensity depends not only on the inherent crystal structure but also on the specimen geometry and the diffraction condition by the dynamical diffraction effect and the inelastic scattering due to the strong interactions with atoms. To avoid the complexity due to the inelastic scattering, the quantitative techniques of electron diffraction have mainly been developed not only on the basis of direct measurement of diffraction intensities but on the observation of phenomena caused by the strong dynamical effect: thickness fringes, CBED rocking curves, the critical voltage effect and the intersecting Kikuchi line (IKL) ${ }^{(2)}$. CBED has come into wide use with spread of modern analytical electron microscopes, because it provides a great deal of information on crystal symmetry, lattice parameters and orientations, all obtain- able from local regions of specimens ${ }^{(3)-(6)}$. Furthermore the CBED technique contributes to improvements of the accuracy and spatial resolution in structure factor determination by the critical voltage or IKL method ${ }^{(2)}$.

On the other hand, recent developments of energyfilters as well as new recording systems have opened up a new field of quantitative analysis of diffraction intensities of electrons ${ }^{(5)(7)-(10)}$. The energy-filters attached with TEM subtract considerably the inelastic scattering component, and the filtered diffraction patterns are recorded in the new media such as an imaging plate or a slow scan CCD camera which has high sensitivity, a wide dynamic range and good linearity between the input and output signals, as compared with conventional negative films ${ }^{(11)-(14)}$. Thus the accurate measurement of elastically scattered intensities of electrons has become practical recently ${ }^{(8)}$.

The present paper reviews some applications of electron diffraction techniques to extract quantitative information in materials science with a strong focussing on the CBED technique. We also discuss future prospects of electron diffraction in relation to energy-filtering transmission electron microscopy.

\section{The Critical Voltage and the Intersecting Kikuchi Line Methods}

\section{The critical voltage effect}

The critical voltage effect appears under a condition of excitation of systematic reflections ${ }^{(15)(16)}$ as well as nonsystematic excitation or under zone-axis incidence ${ }^{(17)-(20)}$. 
The critical voltage effect is observed in electron diffraction patterns as disappearing of a reflection or reversal of asymmetric contrast for relevant diffraction at a certain accelerating voltage (or the critical voltage). One of examples is shown in Fig. 1, where the 440 systematic critical voltage effect in a $\mathrm{Si}-71$ at $\% \mathrm{Ge}$ alloy is given ${ }^{(21)}$. When the accelerating voltage is changed under the exact 440 Bragg condition, the intensity of 440 reflection takes a minimum at $1066 \mathrm{kV}$, and the asymmetric contrast of the middle line at 220 reflection reverses above and below $1066 \mathrm{kV}$. The critical voltage effect is explained in terms of the degeneracy of the main two branches of the dispersion surface due to the relativistic effect and the many-
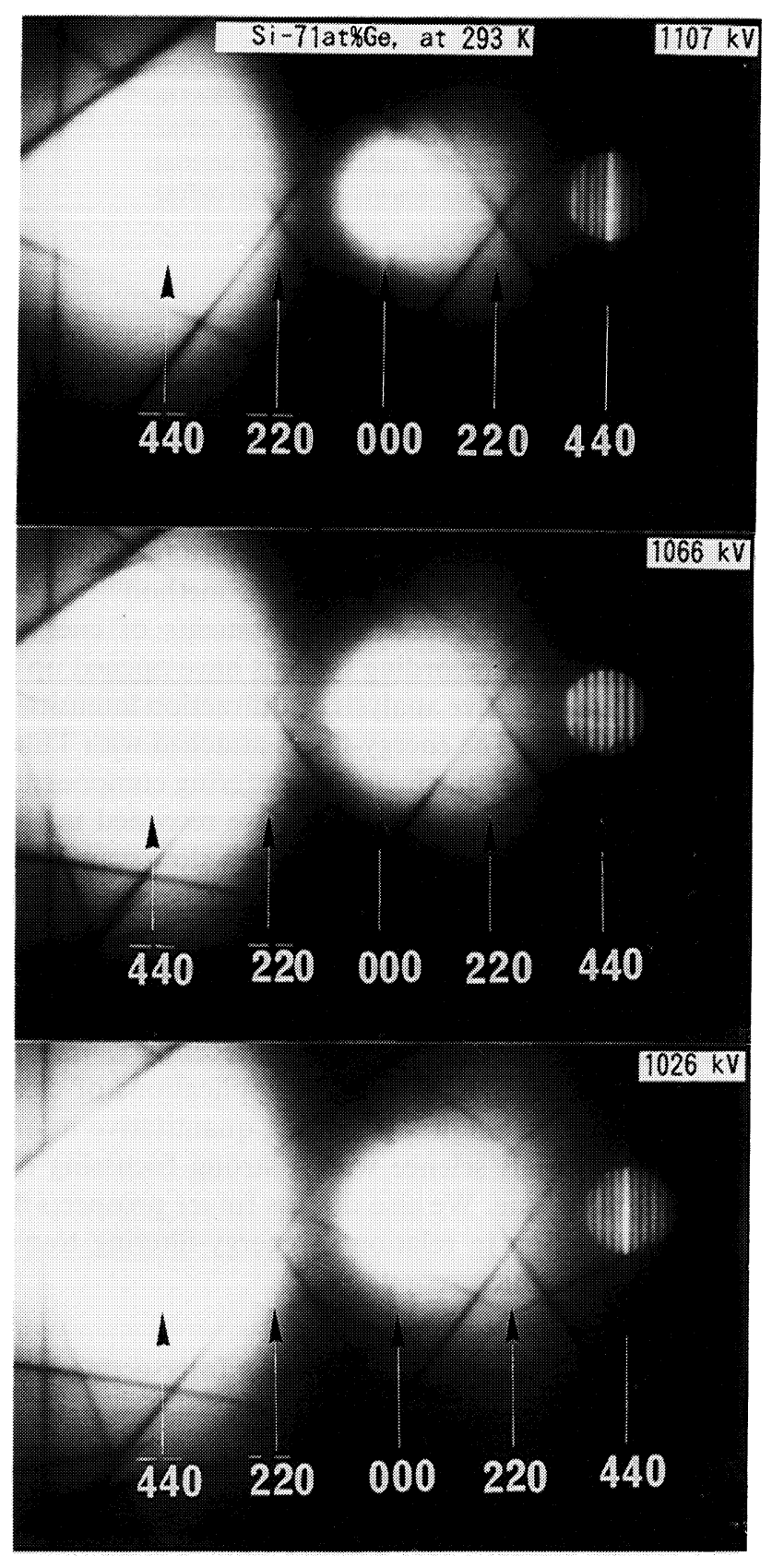

Fig. 1 Critical voltage effect of 440 systematic reflection in Si-71 at $\% \mathrm{Ge}$ at room temperature. beam dynamical scattering of fast electrons ${ }^{(15)(16)}$. According to the three-beam approximation of systematic reflections $0, \boldsymbol{h}$ and $2 \boldsymbol{h}$, the critical voltage is given by

$$
V_{\mathrm{c}}=\frac{m_{0} c}{e}\left(\frac{U(2 h) h^{2}}{U(\boldsymbol{h})^{2}-U(2 h)^{2}}-1\right),
$$

where $U(\boldsymbol{h})$ and $U(2 \boldsymbol{h})$ are the structure factors of $\boldsymbol{h}$ and $2 h$ reflections, $m_{0}, c$ and $e$ have the usual meanings used in electron diffraction. Thus the value of $V_{\mathrm{c}}$ depends on low order structure factors. As the structure factor is given as

$$
U(h)=\sum_{i} f_{\mathrm{i}}(h) T_{\mathrm{i}}(h) \exp \left(2 \pi i h r_{\mathrm{i}}\right)
$$

one can accurately determine a low order atomic scattering factor $f(h)$ and/or a temperature factor $T(h)$ by measuring the critical voltage. The critical voltage depends on specimen temperature through $T(h)$ which is related to the atomic mean square displacement $\left\langle u^{2}\right\rangle$. Values of the measured critical voltages of $\mathrm{Si}$ and $\mathrm{Ge}$ change linearly with temperature as shown in Fig. $2^{(22)}$. The isolated atom potential in a crystal with cubic symmetry is given as a function of displacement $\boldsymbol{u}=\left(u_{1}, u_{2}, u_{3}\right)^{(23)}$ :

$$
v(u)=v_{0}+\frac{1}{2} \alpha u^{2}+\beta u_{1} u_{2} u_{3}+\gamma u^{4}+\cdots .
$$

Here $u^{2}=u_{1}^{2}+u_{2}^{2}+u_{3}^{2}$. The third term in eq. (3) is due to the antisymmetric contribution that appears in a diamond-type lattice, where the center of inversion symmetry is displaced from atom sites. The fourth and higher terms represent anharmonic components of the potential. If one tries to explain the temperature dependence of $V_{\mathrm{c}}$ given in Fig. 2 with eq. (3), one obtains $\alpha(\mathrm{Si})$ $=7.5 \pm 1.5, \quad \alpha(\mathrm{Ge})=5.73 \pm 0.38 \quad\left[10^{-17} \mathrm{~J} / \mathrm{nm}^{2}\right], \quad$ and negligibly small third and fourth terms. The curves in Fig. 2 are calculated with the first and second terms only, or with the harmonic approximation of atom vibration.

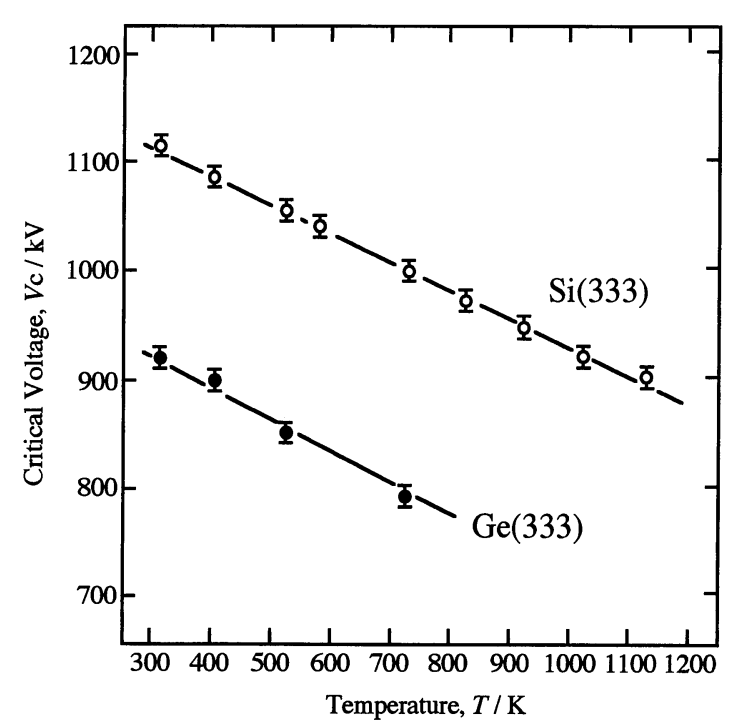

Fig. 2 Critical voltages measured as a function of temperature for $\mathrm{Si}$ and $\mathrm{Ge}$ under excitation of systematic 333 reflection. 
The good agreement between the measured and calculated results indicates that the effect of anharmonic vibration is very weak for $\mathrm{Si}$ and $\mathrm{Ge}$ in the temperature range $300-1078 \mathrm{~K}^{(22)}$. In contrast, the anharmonic effect of vibration is significant in metals with the cubic lattice such as $\mathrm{Al}, \mathrm{Cu}$ and $\mathrm{Fe}^{(23)}$. The observed temperature dependence of the critical voltages for these metals are compared in Fig. 3 with the calculations based on harmonic, quasi-harmonic and anharmonic approximations. The quasi-harmonic approximation takes account of the effect of thermal expansion on the harmonic vibration using the temperature dependent $\alpha$, but neglects the third and fourth terms in eq. (3). The thermal vibration in $\mathrm{Fe}$ can be recognized as quasi-harmonic, while the anharmonic behavior is significant in the other metals.

Thus the critical voltage effect is quite useful for determination of $f(\boldsymbol{h})$ and $T(\boldsymbol{h})$, but the applicable field has been limited because the measurements of the critical voltages sometimes need a high voltage electron microscope $^{(2)(24)}$ and the measurable critical voltage does not always exist. The critical voltage effect has also been recently found under many-beam conditions, such as of non-systematic excitation and of zone-axis incidence. The value of $V_{\mathrm{c}}$ becomes usually lower in the latter cases than under a simple systematic condition. A conventional $200 \mathrm{kV}$ electron microscope or a medium voltage

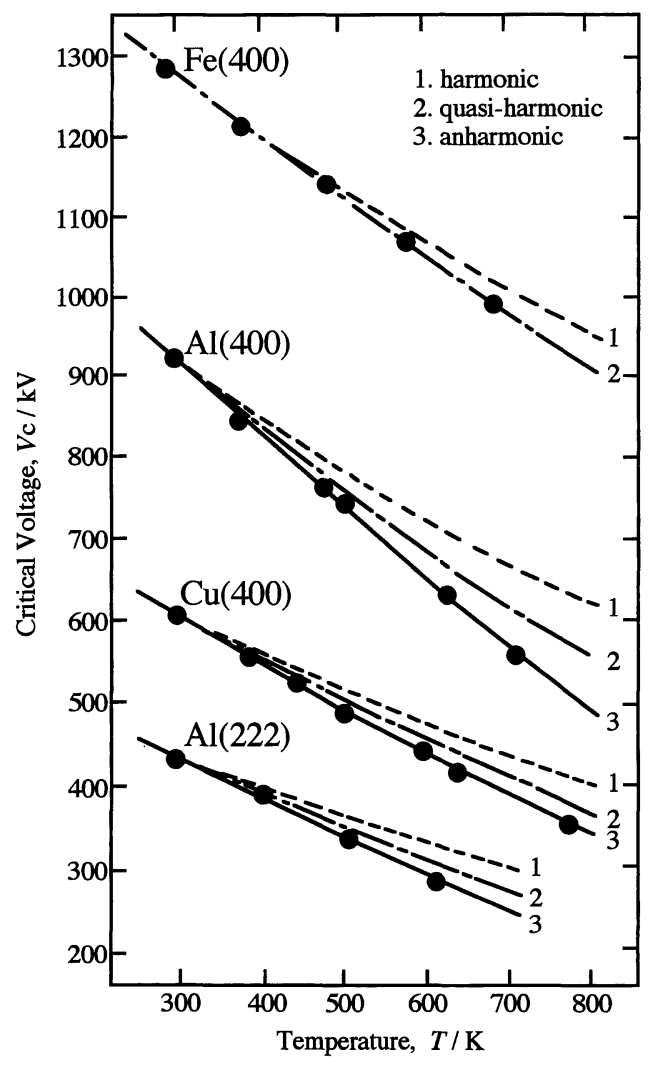

Fig. 3 Temperature dependence of the systematic critical voltages for $\mathrm{Al}, \mathrm{Cu}$ and $\mathrm{Fe}$. Broken, dash-dot, and full curves are the calculations based on harmonic, quasi-harmonic, and anharmonic approximations, respectively. machine of 300 or $400 \mathrm{kV}$ is usable for the measurement of many-beam critical voltage. One of the examples is given in Fig. 4, which shows the [110] zone axis critical voltage effect of $\mathrm{Si}^{(25)}$. Intensities indicated by arrows in the 000 or 002 disc are nearly equal at $147 \mathrm{kV}$ and they are reversed above and below $147 \mathrm{kV}$. Thus CBED is of great help for observation of the zone axis critical voltage effect.

Figure 5 shows [001] zone axis CBED patterns taken from different regions of $\mathrm{YBa}_{2} \mathrm{Cu}_{3} \mathrm{O}_{y}$ that includes small particles of $\mathrm{Y}_{2} \mathrm{BaCuO}_{5}(211 \text { phase })^{(26)}$. The zone axis critical voltage at point $A$ indicated in Fig. 5 (c) is $178 \pm 2 \mathrm{kV}$, while the one at point $B$ is $184 \pm 2 \mathrm{kV}$. The calculated value of the zone axis critical voltage also changes linearly with oxygen concentration $y$ as shown in Fig. 6. The agreement between the measured and the calculated results is unsatisfactory, probably because of uncertainty in values of $f(h)$ 's used in the calculation. However the

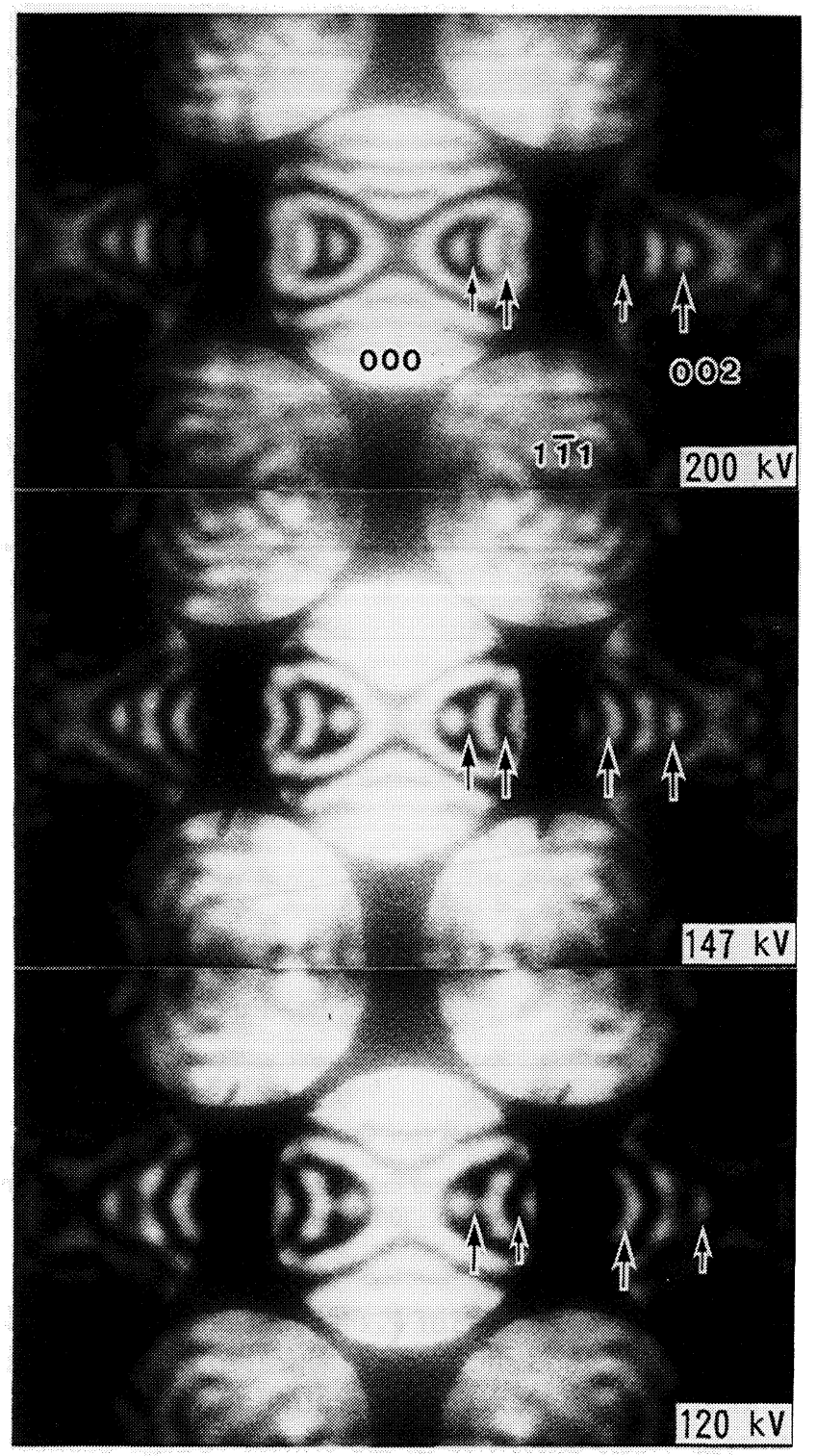

Fig. 4 Zone-axis critical voltage effect of Si along [110] at room temperature. 

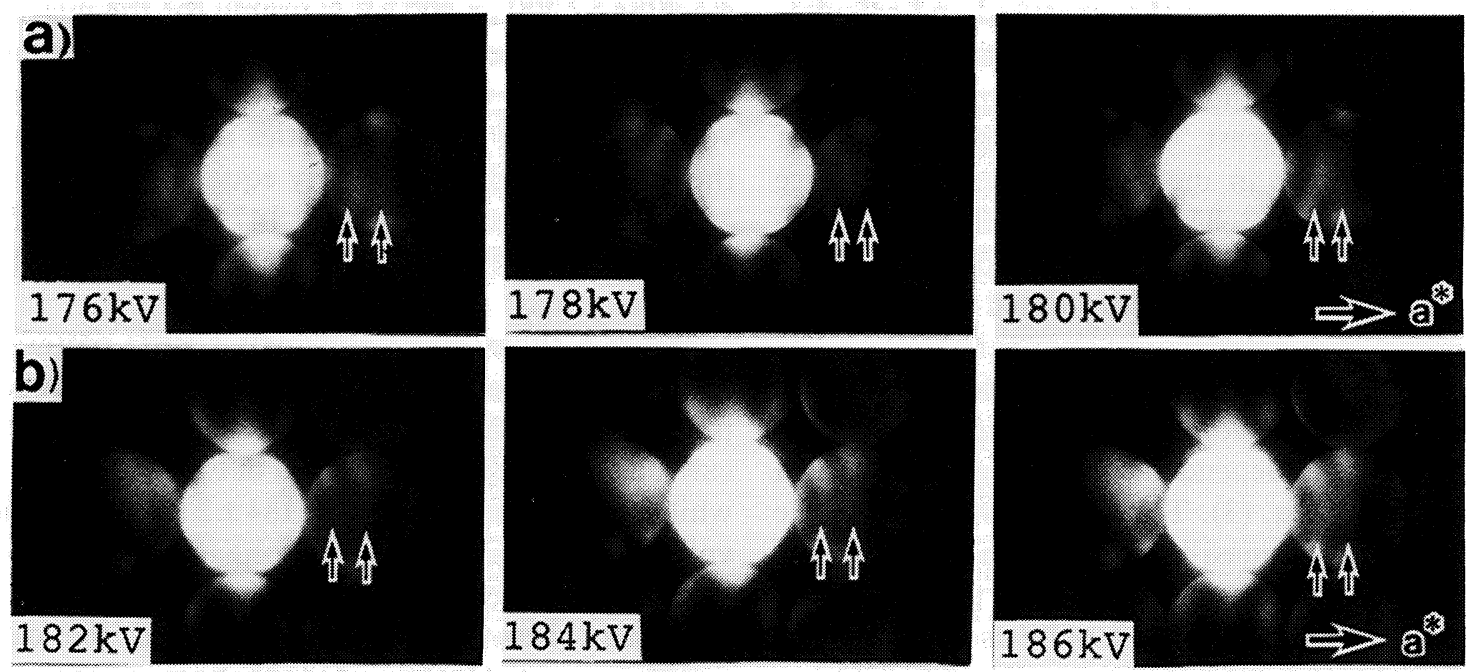

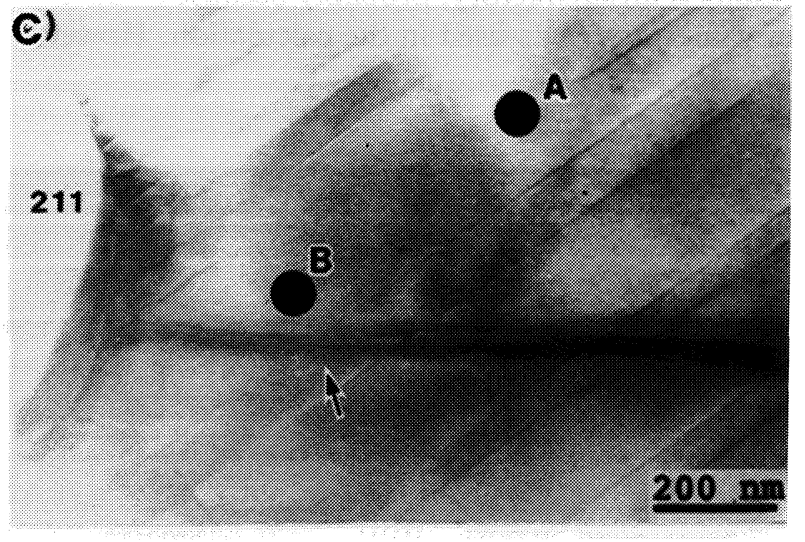

Fig. $5[001]$ zone axis critical voltage effect of $\mathrm{YBa}_{2} \mathrm{Cu}_{3} \mathrm{O}_{y}(\mathrm{a}, \mathrm{b})$ and bright-field image (c) showing twin lamellas, a particle of 211-phase and a dislocation. (a) and (b) were taken at points $\mathrm{A}$ and $\mathrm{B}$, respectively.

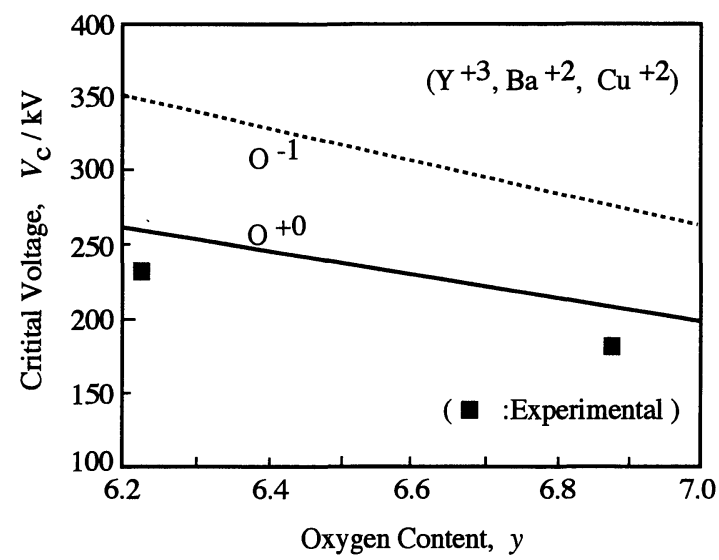

Fig. 6 Zone-axis critical voltages calculated as a function of oxygen concentration $y$ in $\mathrm{YBa}_{2} \mathrm{Cu}_{3} \mathrm{O}_{y}$. [001] axis, at room temperature.

almost linear dependence of $V_{\mathrm{c}}$ on $y$ indicates that the critical voltage method should be practical to detect relative or local change in oxygen concentration in the high $T_{\mathrm{c}}$ materials. The difference in the critical voltage between points $\mathrm{A}$ and $\mathrm{B}$ is due to a local change in oxygen concentration, $\Delta y=0.078 \pm 0.058$.

\section{Intersecting Kikuchi line method}

The structure factor $U(\boldsymbol{h})$ can also be determined by the intersecting Kikuchi line method ${ }^{(27)(28)}$. A Kikuchi line (or Kossel line in a CBED disc) of a high order reflection $\boldsymbol{g}$ splits at an intersection point with a low order reflection $\boldsymbol{h}$ on account of dynamical interaction. One of the examples is given in Fig. 7 where a CBED pattern of the $\mathrm{Cu}-$ $\mathrm{Au}-\mathrm{Pd}$ alloy with an $L 1_{0}$ type superlattice is shown ${ }^{(29)}$. The Kikuchi lines of 646 and 647 reflections that satisfy the Bragg conditions split at intersections with a Kikuchiband of 001 reflection. The CBED technique is useful since the Kossel lines clearly appear in a CBED disc even if the Kikuchi lines are difficult to observe ${ }^{(30)}$. The distance $D(h)$ of the split Kikuchi lines sensitively depends on the low order structure factor $U(h)$ as apparent from the following equation derived with a three-beam approximation with $0, \boldsymbol{g}$ and $\boldsymbol{g}+\boldsymbol{h}$ reflections,

$$
D(\boldsymbol{h})=\frac{U(\boldsymbol{h})}{g}\left(1+\frac{e E}{m_{0} c^{2}}\right),
$$

where $g$ is the magnitude of $\boldsymbol{g}$, and $E$ is the accelerating voltage. The intersecting Kikuchi line method has less limitation in the index $\boldsymbol{h}$ and in the accelerating voltage, in contrast to the critical voltage method. The value of $D(\boldsymbol{h})$ increases with the accelerating voltage. Consequently, the accuracy of measurement of $D(h)$ is improved when a CBED pattern is taken at a high accelerating voltage. The values of $V_{\mathrm{c}}$ and $D(\boldsymbol{h})$ depend on low order structure factors in different manners as can be known from eqs. (1) and (4). Hence one can simultaneously determine both $f(h)$ and $T(h)$ measuring $V_{\mathrm{c}}$ and $D(\boldsymbol{h})$ at a given temperature ${ }^{(22)(31)}$. As an example, Fig. 8 demonstrates simultanenous determination of $f(220)$ and $B$-factor for $\mathrm{Ge}^{(22)}$. Here the latter factor defines $T(\boldsymbol{h})$ as $T(h)=\exp \left(-B h^{2} / 4\right)$ in the harmonic approximation.

The intersecting Kikuchi line method is very useful for a study of the atomic configuration in ordered ternary alloys when it is combined with the ALCHEMI (Atom Location by Electron Channeling Enhanced X-ray Microanalysis). Examples will be given in a later section. 

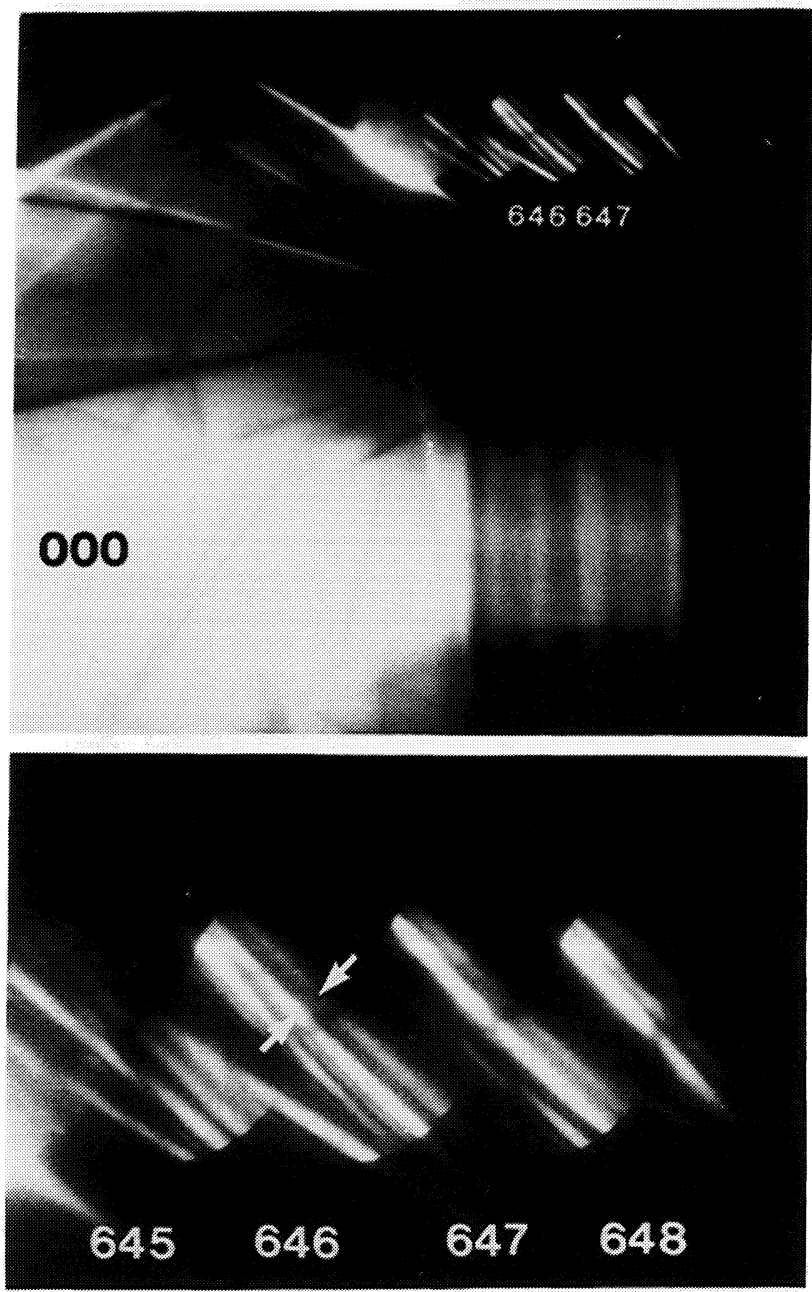

Fig. 7 IKL pattern of $\mathrm{Cu}_{52} \mathrm{Au}_{37} \mathrm{Pd}_{11}$ observed at $200 \mathrm{kV}$. The whole pattern (upper) and the enlarged one (lower). The 646 and 647 reflections satisfy simultaneously the exact Bragg condition. White arrows indicate the splitting of 646 line due to 001 reflection.

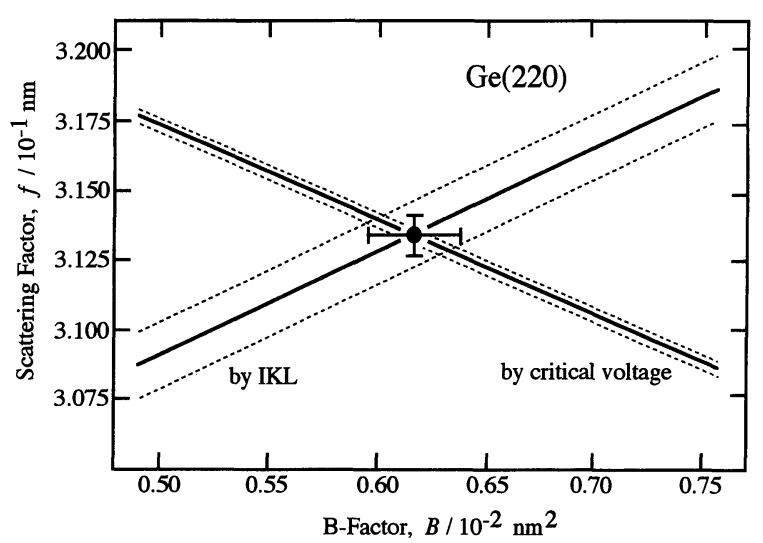

Fig. 8 Simultaneous determination of $f(220)$ and $B$ of Ge. Lines show relations between $f$ and $B$ obtained by the measured critical voltage and the separation of Kikuchi lines.

\section{Local Lattice Parameters by CBED}

CBED provides various kinds of information necessary for the characterization of crystalline materials: determination of crystal symmetries, characterization of a dislocation and a stacking fault, measurement of structure factors and so on ${ }^{(6)}$. We will demonstrate the usefulness of CBED for the measurement of local lattice parameters or lattice strains. A set of higher order Laue zone (HOLZ) lines appear in a bright-field disc of zone-axis CBED pattern. The geometry of HOLZ lines is very sensitive to a change in the deviation parameter $s$, or lattice parameters. Figure 9 shows an example of [012] HOLZ patterns of Si taken at 300 and $100 \mathrm{~K}$. The crossing points of HOLZ lines shift with cooling the specimen as indicated by arrows in Fig. 9. This line shift is ascribed to a change in lattice parameter, because all electron-optical conditions are fixed. The change in the lattice parameter can be detected by matching observed HOLZ patterns with simulated ones with the kinematical approximation of diffraction. According to the analysis of Fig. 9, the
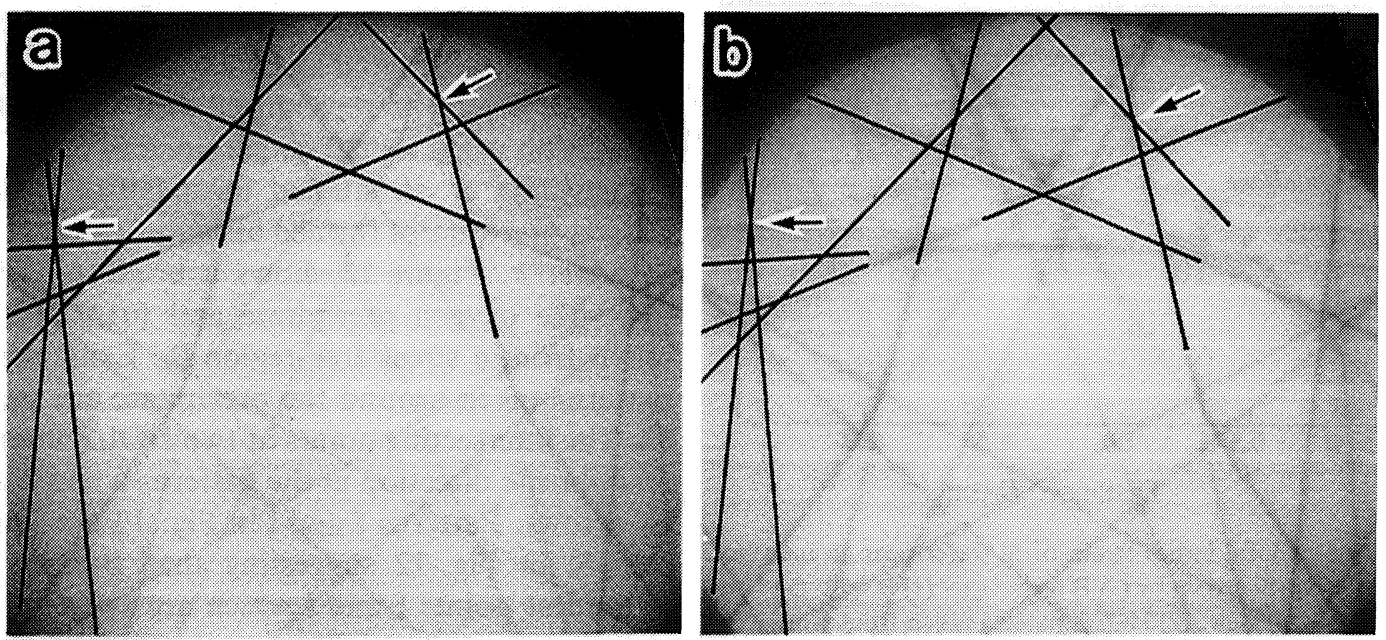

Fig. 9 Change of [012] HOLZ patterns in Si with temperature. (a) $300 \mathrm{~K}$, (b) $100 \mathrm{~K}$ at $200 \mathrm{kV}$. 
change in the lattice parameter is estimated to be $0.05 \%$ which is in good agreement with the value predicted by the thermal expansion coefficient of Si. We can detect a change as small as $0.01 \%$ in lattice parameters as demonstrated in Fig. 10 ${ }^{(32)}$. [012] HOLZ patterns observed near an interface between poly-Si and Si substrate have a mirror symmetry with respect to a line $m-m^{\prime}([0 \overline{2} 1]$ axis) and no significant difference in appearance. The observed HOLZ patterns are well matched with a tetragonally distorted lattice. A local lattice distortion $\Delta a / a_{0}$ ( $a$-axis is perpendicular to the interface) is obtained by comparing measured A/B with the one simulated as a function of the tetragonality.

The kinematical approximation is simple and of easy handling in the pattern simulation, but one should pay attention to the dynamical diffraction effect when the absolute values of lattice parameter is measured ${ }^{(33)(34)}$. The observed HOLZ lines often shift systematically from the positions predicted by the kinematical approximation because of the dynamical effect of electron diffraction. Double lines appear in certain cases for one HOLZ reflection and the visibility of the two lines varies with the composition and thickness of the specimen. These phenomena caused by the dynamical diffraction effect are explained in terms of dispersion surfaces as depicted in Fig. 11. In the kinematical theory, a HOLZ line for reflection $G$ is expected to arise from intersection (P in Fig. 11) of two spheres of radius $K$ centered on the origin $\mathrm{O}$ and point G, respectively. The HOLZ line would appear at the position given by $\mathrm{PO}$, where $\triangle \mathrm{POG}$ is an isosceles
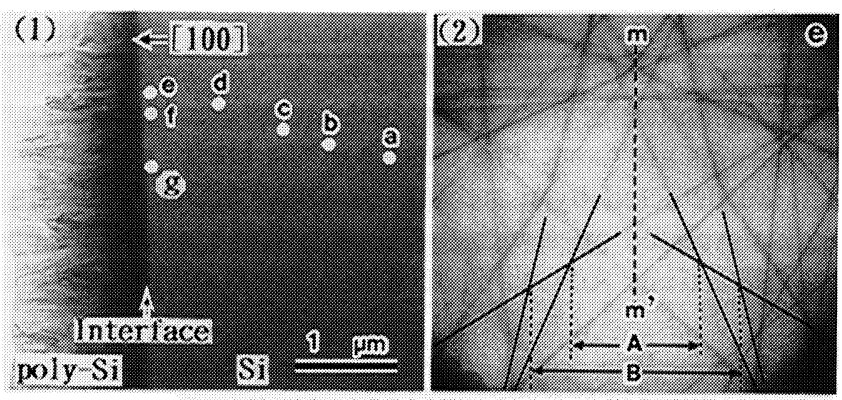

(3)

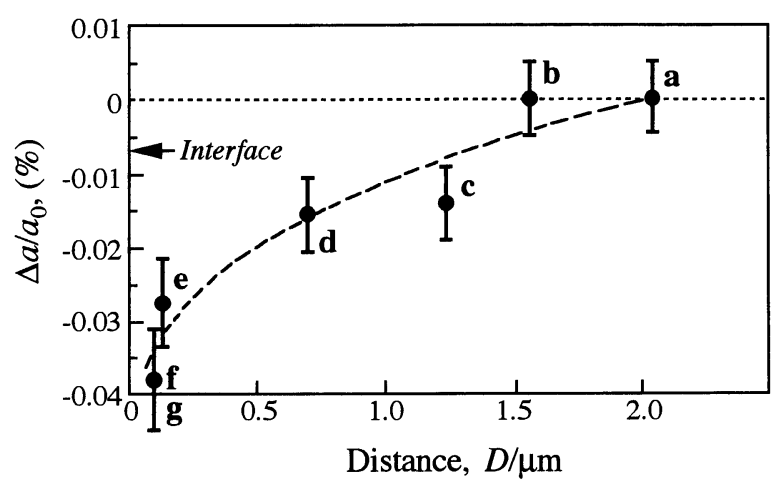

Fig. 10 Detection of local lattice strain of Si wafer near an interface between $\mathrm{Si}$ and poly-Si grown by CVD technique. (1) Bright-field image of cross-sectional view, (2) HOLZ pattern taken from point e indicated in (1). (3) Lattice strains measured as a function of distance from the interface.

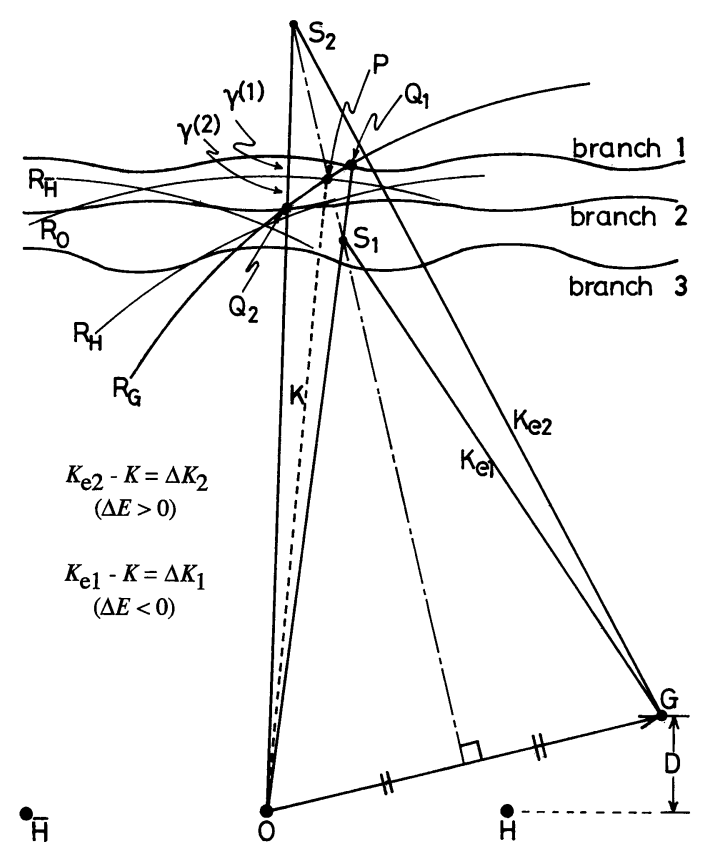

Fig. 11 Schematic diagram of dispersion surface to explain the dynamical effect upon positions of HOLZ lines and the origin of $\Delta E$,

triangle as apparent in Fig. 11. A HOLZ line is produced actually from an intersection of the sphere of radius $K$ (centered on $\mathrm{G}$ ) with a branch of the zeroth-order dispersion surface. Therefore, plural HOLZ lines may be generated for each reflection. If a HOLZ reflection $\boldsymbol{G}$ is caused by Bloch wave 1 , or branch 1 , which is situated above the sphere of radius $K$ by $\gamma^{(1)}$, the position of the line is given by $\mathrm{Q}_{1} \mathrm{O}$ in stead of $\mathrm{PO}$. In order to explain the line at the position $\mathrm{Q}_{1} \mathrm{O}$ on the basis of the kinematical theory, we have to set an apex of the isoceles triangle at the point $S_{1}$ which lies on the line $O Q_{1}$. The wave number necessary for the pattern simulation is not $K$ but $K_{\text {el }}$ which is smaller than $K$ by $\Delta K_{1}^{(35)} . \Delta K_{\mathrm{j}}$ is converted to a change in accelerating voltage $\Delta E$ and related to the eigen value $\gamma^{(j)(36)}$. If we use the effective electron energy $E_{\mathrm{e}}$ in stead of the actual microscope operating voltage $E_{\mathrm{a}}$, where $\Delta E=E_{\mathrm{e}}-E_{\mathrm{a}}$, the kinematical simulation is applicable to the accurate determination of lattice parameters. $E_{\mathrm{e}}$ is so determined that an observed HOLZ pattern of the reference crystal is reproduced well by the kinematical simulation using its lattice parameters ${ }^{(37)}$.

Okuyama et al. studied local lattice strains around oxygen precipitates formed as impurity gettering sinks in $\mathrm{Si}$ wafer $^{(38)}$. Figure 12 shows a bright-field image of a plate-like oxygen precipitate and [012] HOLZ pattern taken from a stress free region $\mathrm{S}_{\mathrm{f}}$. Examples of HOLZ patterns taken around the precipitate are shown in Fig. 13. The intersecting points of two HOLZ lines shift along an opposite direction in the patterns $\mathrm{a}$ and $\mathrm{h}$ as indicated by arrows. Patterns $f$ and $l$ in Fig. 13 are similar to pattern $\mathrm{S}_{\mathrm{f}}$ in Fig. 12 suggesting that stresses are almost relaxed at points $f$ and 1 which are apart by $2 \mu \mathrm{m}$ from the precipitate. According to a close comparison between the observed and simulated patterns, the lattice strains 


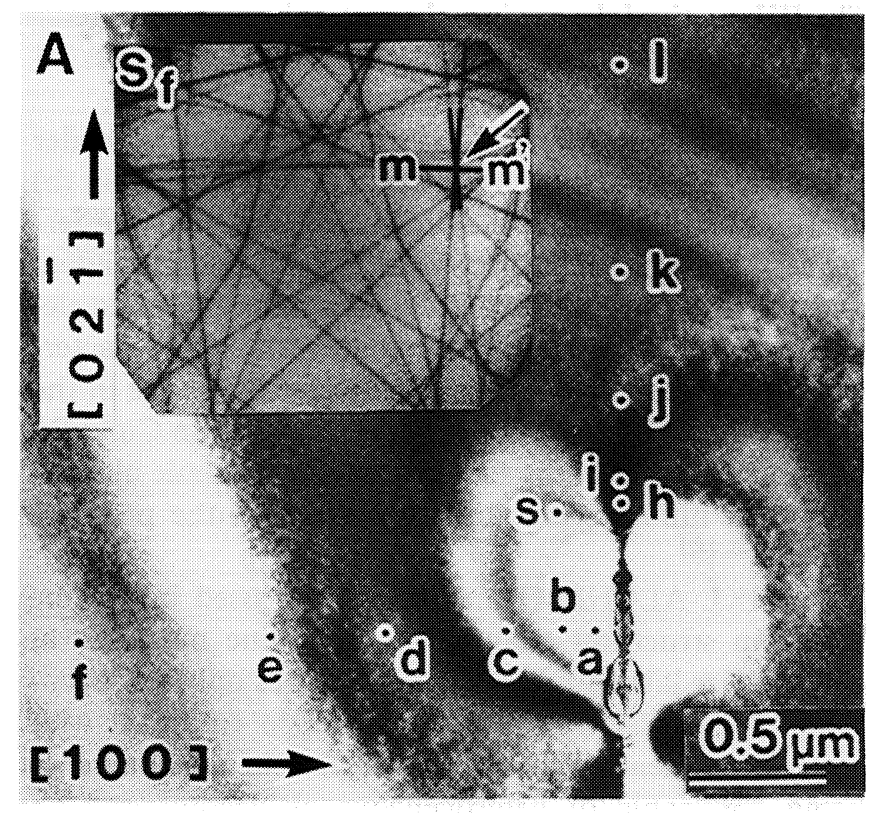

Fig. 12 Bright-field image (A) of a plate-like oxygen precipitate in $\mathrm{Si}$ wafer observed along [012]. The precipitate is parallel to (100) plane of Si. Points indicated by a-f and h-l show positions where HOLZ patterns are observed.

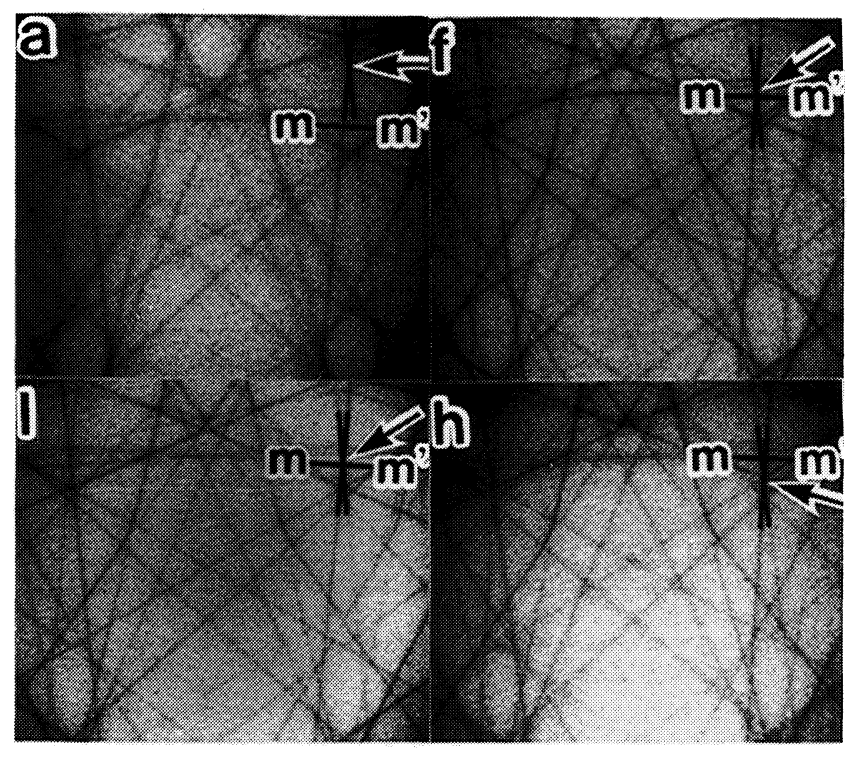

Fig. 13 Examples of [012] HOLZ patterns taken from points indicated in Fig. 12.

are tensile along a-f and compressive along h-i as shown in Fig. 14.

\section{IKL and ALCHEMI}

As discussed in a previous section, the critical voltage and the IKL methods enable us to measure the structure factor of a reflection with sufficient precision. However the former technique is mostly unavailable to a superlattice reflection $\boldsymbol{h}$, owing to the fact that the magnitude of $U(h)$ would be generally smaller than $U(2 h)$ for the

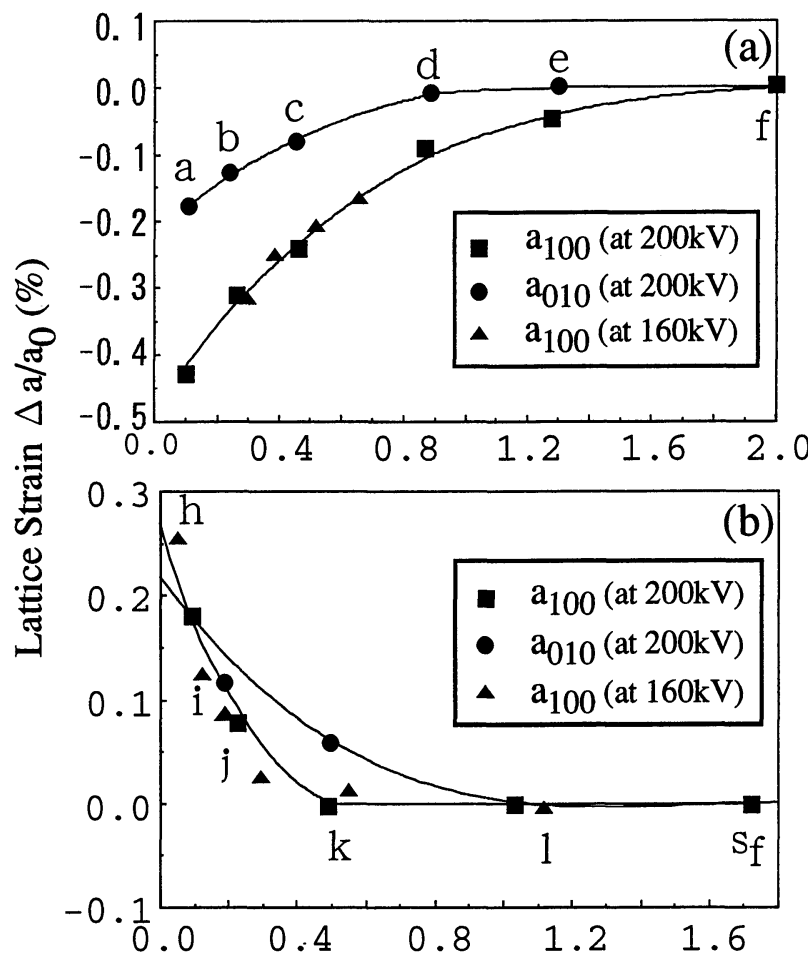

Distance from the Precipitate Interface,$X / \mu \mathrm{m}$

Fig. 14 Lattice strains measured as a function of distance from the oxygen precipitate shown in Fig. 12.

nearest fundamental reflection. It follows a negative value of $V_{\mathrm{c}}$ for a superlattice reflection, as seen in eq. (1). The IKL method is useful to determine atomic configuration in an ordered compound, since the separation of Kikuchi line $D(h)$ is directly proportional to $U(h)$ of the relevant reflection. One can evaluate the structure factor of 001 superlattice reflection of $L 1_{0}$-type ordered $\mathrm{Cu}$ AuPd from the splitting indicated by arrows in Fig. 7. Matsuhata et al. measured degree of order in $\mathrm{Cu}_{3} \mathrm{Au}$ by IKL ${ }^{(30)}$ However, one would often meet another difficulty for ternary or multinary alloys, where plural order parameters should be used to describe the ordered atomic configuration. For the example of $L 1_{0}$ in $\mathrm{CuAuPd}$ given in Fig. 7, the structure factor of a superlattice reflection is expresed as

$$
U(\boldsymbol{h})=\sum_{i} 2 f_{\mathrm{i}}(\boldsymbol{h}) T_{\mathrm{i}}(\boldsymbol{h}) c_{\mathrm{i}} S_{\mathrm{i}}, \quad i=\mathrm{Cu}, \mathrm{Au}, \text { or } \mathrm{Pd},
$$

where $c_{\mathrm{i}}$ 's are compositions. The long-range order parameters $S_{\mathrm{i}}$ define the occupation probabilities of the elements in the $\alpha$ and $\beta$ sublattices as

$$
\Gamma_{\mathrm{i}}(\alpha)=c_{\mathrm{i}}\left(1+S_{\mathrm{i}}\right) \text {, and } \Gamma_{\mathrm{i}}(\beta)=c_{\mathrm{i}}\left(1-S_{\mathrm{i}}\right) .
$$

The number of independent valuables contained in eq. (5) is two for the ternary case, on account of the conservation law $c_{\mathrm{Cu}} S_{\mathrm{Cu}}+c_{\mathrm{Au}} S_{\mathrm{Au}}+c_{\mathrm{Pd}} S_{\mathrm{Pd}}=0$. Hence, a single measurement of the structure factor given in eq. (5) is not enough to determine the order parameters of $S_{\mathrm{i}}$ or the occupation probabilities $\Gamma_{\mathrm{i}}$. To overcome this difficulty, the combined employment of IKL with ALCHEMI (Atom 
Location by Channeling Enhanced Microanalysis) was proposed $^{(29)}$. The ALCHEMI method is well known as a technique to determine location of impurity atoms in crystalline compounds, taking advantage of characteristic X-ray emission from an illuminated area as a function of diffraction condition ${ }^{(39)}$. The standard ALCHEMI procedure is based on an assumption that the host-element atoms form an ideal sublattice, or there are no antisite atoms of the host. Therefore the application of ALCHEMI has been mostly limited to the intermetallic or inorganic compounds where the location of host elements is clearly known. The combination of IKL and ALCHEMI is available to structural analysis of such systems as multi-component alloys with an order-disorder transition and nonstoichiometric multinary compounds, where the above assumption would be broken down.

Figure 15 shows EDX spectra obtained from CuAuPd with $L 1_{0}$-type order. One can recognize that the characteristic X-ray emission from $\mathrm{Au}$ and $\mathrm{Pd}$ is suppressed under strong dynamical excitation of 011 superlattice reflection with a positive deviation from the exact Bragg position in Fig. 15(a), in comparison with a quasi-kinematical condition without strong excitation of reflections in (b). The relative changes of X-ray emission depending on the diffraction condition give ratios of order parameters, according to

$$
P_{\mathrm{i}, \mathrm{Au}} \equiv \frac{S_{\mathrm{i}}}{S_{\mathrm{Au}}}=1-\frac{N_{\mathrm{i}}^{\mathrm{a}} / N_{\mathrm{i}}^{\mathrm{b}}-N_{\mathrm{Au}}^{\mathrm{a}} / N_{\mathrm{Au}}^{\mathrm{b}}}{\sum_{i} c_{\mathrm{i}}\left(N_{\mathrm{i}}^{\mathrm{a}} / N_{\mathrm{i}}^{\mathrm{b}}-N_{\mathrm{Au}}^{\mathrm{a}} / N_{\mathrm{Au}}^{\mathrm{b}}\right)} .
$$

Here, $N_{\mathrm{i}}^{\mathrm{a}}$ and $N_{\mathrm{i}}^{\mathrm{b}}$ are counts of a characteristic X-ray peak of element $i$ under the conditions (a) and (b), respectively. The number of unknown order parameter included in the structure factor is reduced to one if $P_{\mathrm{i} / \mathrm{Au}}$ are evaluated by ALCHEMI, as

$$
U(h)=\sum_{i} 2\left\{f_{\mathrm{i}}(h) T_{\mathrm{i}}(h) c_{\mathrm{i}} P_{\mathrm{i} / \mathrm{Au}}\right\} S_{\mathrm{Au}}
$$

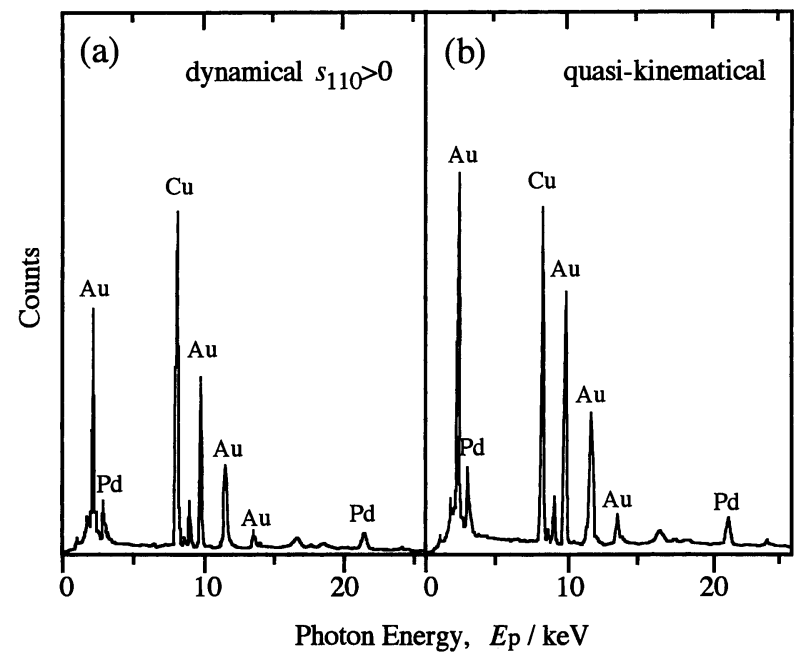

Fig. 15 EDX spectra obtained from $\mathrm{Cu}_{52} \mathrm{Au}_{37} \mathrm{Pd}_{11}$ with $L 1_{0}$-type order. (a) dynamical diffraction condition with $s_{110}>0$, (b) quasi-kinematical condition.
The IKL method determines the rest unknown parameter $S_{\mathrm{Au}}$ through the structure factor measurement. Thus all the order parameters are specified.

Figure 16 demonstrates the time-evolution of order parameters $S_{\mathrm{i}}$ in (a) and occupation probabilities $\Gamma_{\mathrm{i}}$ in (b) in $\mathrm{Cu}_{50} \mathrm{Au}_{35} \mathrm{Pd}_{15}$ during the process of long-range ordering at $573 \mathrm{~K}$ from a disorder state $\mathrm{e}^{(40)(41)}$. It is clearly shown that the ordering rates are different among the three elements, and the occupation probabilities draw a characteristically curved kinetic path of "inverse S" shape in the Gibbs triangle. The behavior of $L 1_{0}$ ordering in CuAuPd has been extensively discussed in terms of atomic interactions and diffusive mobilities of atoms in the previous papers. ${ }^{(40)-(42)}$

The IKL-ALCHEMI method can be used in routine works in structural analysis of crystalline materials at average-size laboratories, since it requires only a conventional transmission electron microscope equipped with an EDX system. This technique has an advantage as being applicable to heterogeneous or decomposed alloys ${ }^{(43)}$.

\section{Structural Refinement by Energy Filtered CBED}

As mentioned in Introduction, the filtering of transmitted and scattered electrons has recently become available
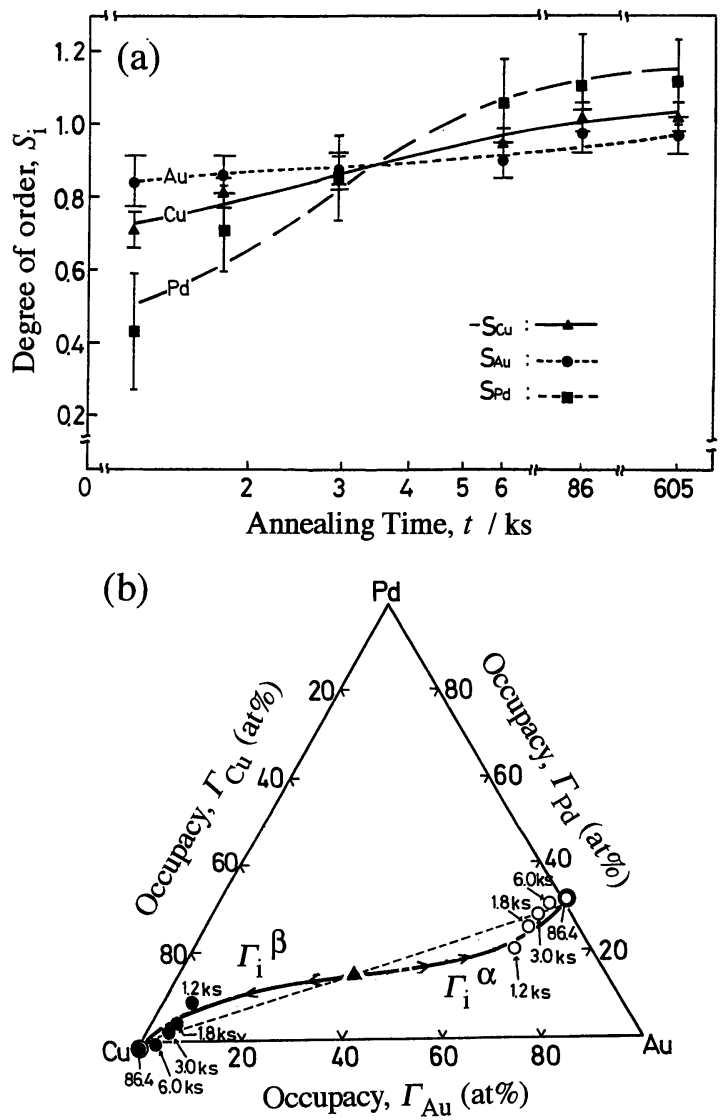

Fig. 16 Temporal change in order parameters $S_{\mathrm{i}}$ in (a) and occupation probabilities $\Gamma_{\mathrm{i}}$ in (b) in $\mathrm{Cu}_{50} \mathrm{Au}_{35} \mathrm{Pd}_{15}$ during the process of longrange ordering at $573 \mathrm{~K}$ from the disorder state. 
in transmission electron microscopy, and has opened up a new field of quantitative analysis of diffraction intensities of electrons. Figure 17 demonstrates the effect of energy-filtering on $\mathrm{CBED}$, where incident electrons were illuminated to $\mathrm{MgO} \cdot n \mathrm{Al}_{2} \mathrm{O}_{3} \quad(n=1.4)$ along the [001] zone axis $^{(44)}$. The filtered pattern (b) was taken with a slit of $\pm 5 \mathrm{eV}$ for zero-loss electrons. One can clearly see that the energy-filtering considerably improves quality of the CBED pattern. It should be noted that weak bright lines are recognized around 200 positions in the filtered pattern (b), but not in the unfiltered one (a). These lines at 200 result from double-reflections with high indices of the first-order Laue zone. If one tries to observe the weak lines under the [001] incidence without an energy-filter, one must carefully tune the electron wave length (or the accelerating voltage) to gain the sufficient intensity ${ }^{(3)}$. The energy-filtering removes sufficiently the diffuse background due to inelastic-scattering, placing the weak reflections in sharp relief at a given accelerating voltage. It should be noticed that the imaging-plate covers over a wide intensity range of CBED without saturation in recording. Thus the combination of energy-filtering and the imaging plate enables us to investigate the details of many-beam dynamical diffraction of elastically scattered electrons. The pattern (b) exhibits no indication of direct reflections of 200. It clearly supports a view that the direct 200 reflections are forbidden and the space group of $\mathrm{MgO} \cdot n \mathrm{Al}_{2} \mathrm{O}_{3}$ spinel is not $F \overline{4} 3 \mathrm{~m}$ but $F \mathrm{~d}_{3} \mathrm{~m}^{(45)(46)}$. Now we are carrying out quantitative analysis of cation displacement in $\mathrm{MgO} \cdot n \mathrm{Al}_{2} \mathrm{O}_{3}$ spinels due to electron or ion irradiation by the filtered CBED. The results will be reported elsewhere ${ }^{(47)}$.

Large angle CBED is useful for structural refinement because one can obtain simultaneously intensity profiles of plural reflections around the exact Bragg positions ${ }^{(48)}$.
Figure 18 shows large angle CBED patterns of $\mathrm{YBa}_{2} \mathrm{Cu}_{3} \mathrm{O}_{y}$ taken under the excitation of 001 systematic reflections. The background intensity is almost eliminated as apparent from the pattern (b) which was taken with the same slit width as above. The intensity profiles read out from recorded imaging plates are also shown in Figs. 18(c) and (d). A slight background in (d) is caused mainly by phonon scattering electrons although the specimen is cooled to $107 \mathrm{~K}$. We compared the observed intensities with those calculated by the many-beam dynamical theory to determine the $z$-coordinates of $\mathrm{Ba}$ atoms in an unit cell. The unit cell of orthorhombic $\mathrm{YBa}_{2} \mathrm{Cu}_{3} \mathrm{O}_{y}$ is composed of three units of a perovskite structure stacked along $z$-direction and a $\mathrm{Ba}$ layer is put between a $\mathrm{CuO}$ chain plane and a $\mathrm{CuO}_{2}$ sheet. Hence the ideal $z$-coordinate of $\mathrm{Ba}$ is $1 / 6$. The obtained value of $z=0.1860 \pm 0.0005^{(49)}$ is different from the ideal value 0.1667 , but in good agreement with the result by neutron diffraction: $0.1863^{(50)}$. This technique is also applicable to the investigation of charge density distribution in compound crystals ${ }^{(5)}$.

\section{Summary}

This paper reviews our recent applications of electron diffraction techniques to quantitative analysis of various kinds of materials. It can be said that the modern electron diffraction has achieved to the stage to derive quantitative and structural information with sufficient accuracy from nanoscale local areas. In particular, the development of CBED has greatly extended the application field of electron diffraction in materials science. The accuracy of quantification is significantly improved by employment of field emission of probe electrons, energy-filtering and new signal detecting or recording systems, which

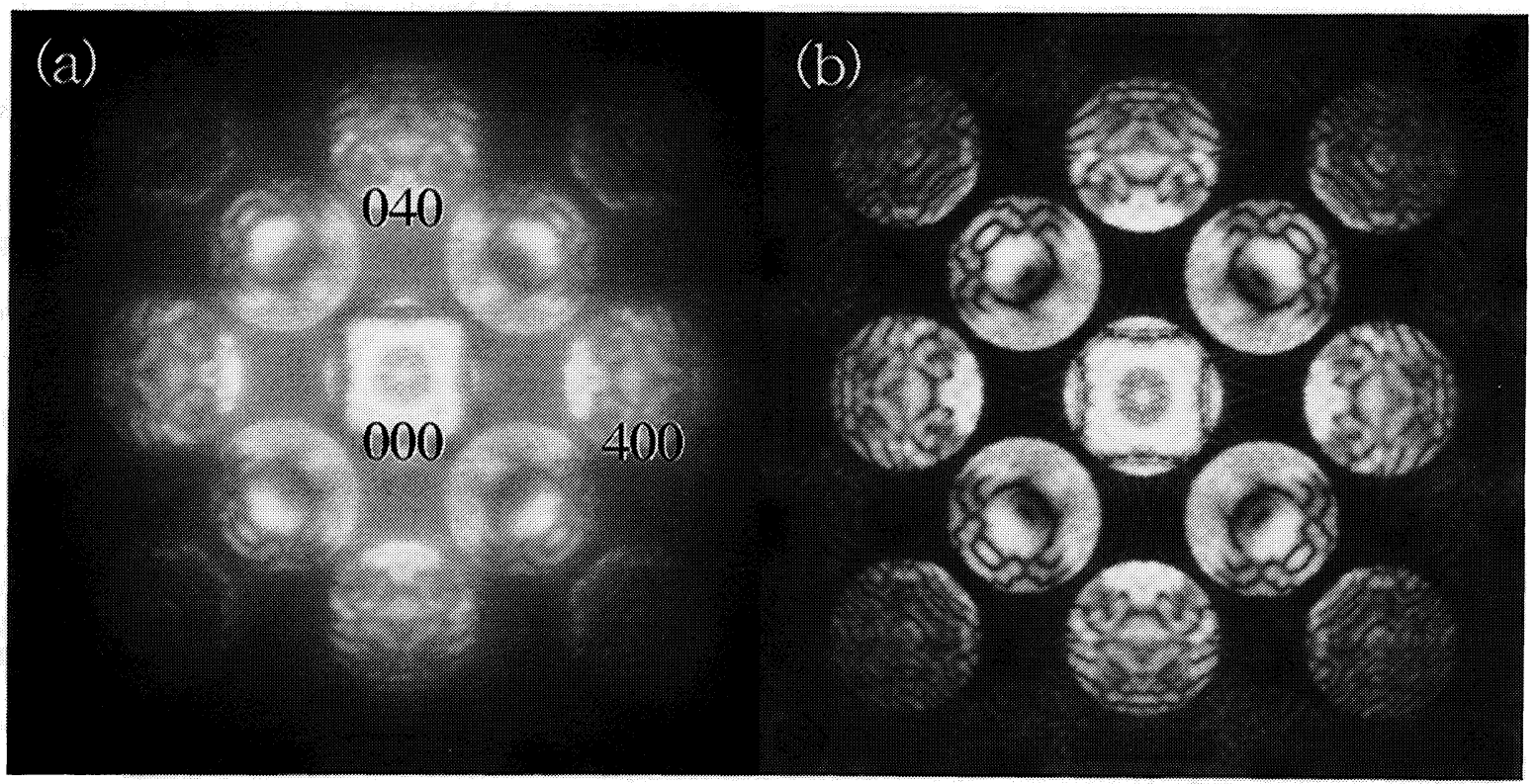

Fig. 17 [001] CBED patterns of $\mathrm{MgO} \cdot n \mathrm{Al}_{2} \mathrm{O}_{3}$ spinel. (a) unfiltered, (b) filtered zero-loss pattern $(-5 \mathrm{eV}<\Delta E<+5 \mathrm{eV})$. $E_{0}=120 \mathrm{kV}, T=107 \mathrm{~K}$. 

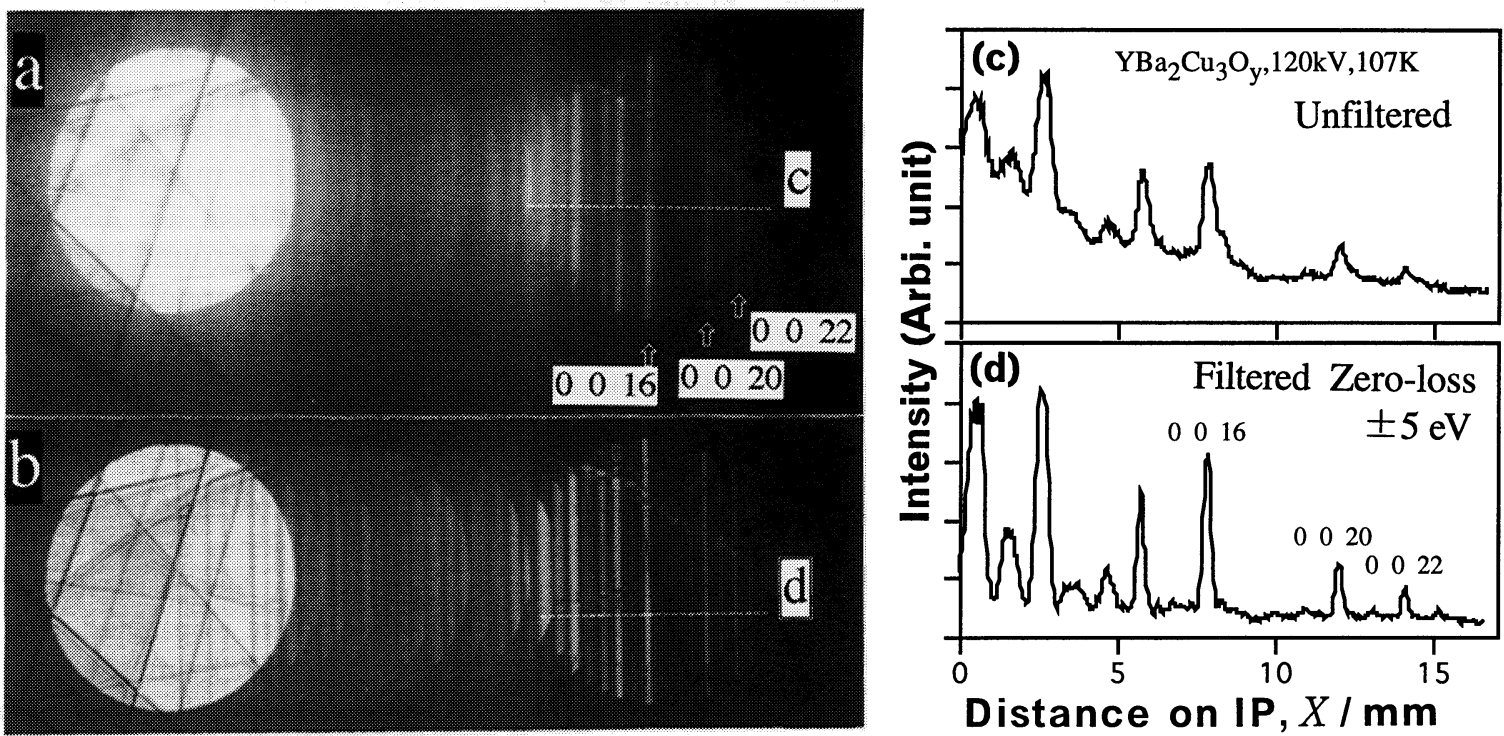

Fig. 18 Observed large angle CBED patterns (a, b) of $\mathrm{YBa}_{2} \mathrm{Cu}_{3} \mathrm{O}_{y}$ and intensity profiles (c, d) read out from imaging plates. (a) unfiltered, (b) filtered $(-5 \mathrm{eV}<\Delta E<+5 \mathrm{eV}) . E_{0}=120 \mathrm{kV}, T=107 \mathrm{~K}$.

have become available recently in analytical electron microscopy. The energy filters are expected to enable us to examine thicker specimens with less thin-foil effects, subtracting inelastically scattered electrons from the diffraction. For example, a HOLZ pattern clearly appears in filtered CBED over a surprisingly wider range of foil thickness. A HOLZ pattern from a thick area should be a function of local lattice dimension almost free from surface relaxation. For this purpose, the combination with a field emission gun and such a high sensitive recording medium as imaging plate or slow-scan CCD camera is effective since the filtering makes the pattern considerably darker. High brightness of a field emission source is also effective to improve the spatial resolution of CBED, EDX and EELS. We believe that the quantitative electron diffraction will become more popular and more versatile in materials science because it is easily combined with some other techniques, such as EDS and EELS, for analyzing composition and electronic states in local areas.

\section{Acknowledgments}

Most of the results demonstrated here were obtained with facilities at the HVEM Laboratory, Kyushu University. The authors should like to express their gratitude to Messrs. E. Tanaka and T. Manabe for their assistance in operation of the microscopes. The work was partly supported by the Grants-in-Aid for scientific research from the Ministry of Education, Science, Sports and Culture, Japan.

\section{REFERENCES}

(1) P. B. Hirsch, A. Howie, R. B. Nicholson, D. W. Pashley and M. J. Whelan: Electron Microscopy of Thin Crystals, Robert E. Krieger, New York, (1977).

(2) Y. Tomokiyo: J. Electron Microsc., 41 (1992), 403.

(3) M. Tanaka and M. Terauchi: Convergent-Beam Electron Diffraction, JEOL-Maruzen, Tokyo, (1985).
(4) M. Tanaka, M. Terauchi and T. Kaneyama: Convergent-Beam Electron Diffraction II, JEOL-Maruzen, Tokyo, (1988).

(5) J. C. H. Spence and J. M. Zuo: Electron Microdiffraction, Plenum Press, New York, (1992).

(6) M. Tanaka, M. Terauchi and K. Tsuda: Convergent-Beam Electron Diffraction III, JEOL, Tokyo, (1994).

(7) J. C. H. Spence: Acta Cryst., A49 (1993), 231.

(8) J. Mayer, C. Deininger and L. Reimer: Energy-filtering Transmission Electron Microscopy, Ed. by L. Reimer, Springer-Verlag Berlin, (1995), p. 291.

(9) Y. Tomokiyo, S. Matsumura and T. Manabe: Ann. Rep. HVEM Lab. Kyushu Univ., 21 (1997), 3.

(10) L. Reimer: This special issue of Mater. Trans. JIM, (1998).

(11) D. Shindo, K. Hiraga, T. Oikawa and N. Mori: J. Electron Microsc., 39 (1990), 449.

(12) Y. Tomokiyo: Ann. Rep. HVEM Lab. Kyushu Univ., 19 (1995), 3.

(13) J. M. Zuo, M. R. McCartney and J. C. H. Spence: Ultramicroscopy, 66 (1996), 35.

(14) A. Taniyama, D. Shindo and T. Oikawa: J. Electron. Microsc., 46 (1997), 303.

(15) F. Nagata and A. Fukuhara: Jpn. J. Appl. Phys., 6 (1967), 1233.

(16) D. Watanabe, R. Uyeda and A. Fukuhara: Acta Cryst., A25 (1969), 138.

(17) H. Matsuhata and J. Steeds: Philos. Mag. A, 55 (1986), 39.

(18) H. Matsuhata and J. Gjønnes: Acta Cryst., A50 (1994), 107.

(19) H. Matsuhata, J. Gjønnes and J. Taftø: Acta Cryst, A50 (1994), 115.

(20) H. Matsuhata and J. Gjønnes: Acta Cryst., A52 (1996), 686.

(21) T. Eguchi, Y. Tomokiyo and H. Matsuhata: J. Microsc. Spectrosc. Electron., 12 (1987), 559.

(22) S. Matsumura, Y. Tomokiyo and K. Oki: J. Electron Microsc. Tech., 12 (1989), 262.

(23) B. T. M. Willis and A. W. Pryor: Thermal Vibrations in Crystallography, Cambridge Univ. Pr., London, (1975).

(24) Y. Tomokiyo, Y. Omori, T. Yoshino and T. Fujimoto: Proc. VIth China-Japan Electron Microscopy Seminar, Okayama, Nakanishi Printing Co. Ltd., Kyoto, (1991), p. 23.; Proc. 10th Euro. Cong. Electron Microsc. Vol. 2, Ed. by A.Lopez-Galindo, MI. Rodriguez-Gracia, Univ. Granada, (1992), p. 157.

(25) Y. Tomokiyo, T. Yoshino and T. Kuroiwa: Proc. Fifth ChineseJapanese Electron Microscopy Seminar, Springer-Verlag, (1990), p. 97.

(26) Y. Tanaka, T. Fujimoto, Y. Tomokiyo and Y. Suyama: Advances in Superconductivity VII, Ed. by K. Yamafuji and T. Morishita, 
Springer Verlag Tokyo, (1994), p.531. and Proc. VIIIth ChinaJapan Electron Microscopy Seminar, Nakanishi Printing Co. Ltd., Kyoto, (1995), p. 37.

(27) J. Gjønnes and R. Høier: Acta Cryst., A27 (1971), 313.

(28) O. Terasaki, D. Watanabe and J. Gjønnes: Acta Cryst., A35 (1979), 895.

(29) S. Matsumura, T. Morimura and K. Oki: Mater. Trans., JIM, 32 (1991), 905.

(30) H. Matsuhata, Y. Tomokiyo, H. Watanabe and T. Eguchi: Acta Cryst., B40 (1984), 544.

(31) S. Matsumura, T. Ohboshi, K. Oki and Y. Tomokiyo: Ultramicroscopy, 39 (1991), 65.

(32) Y. Tomokiyo, J. Nakashima, T. Fujimoto, T. Okuyama, S. Sadamitsu, S. Sumita and T. Shigematsu: Proc. 13th Int. Conf. on Electron Microscopy, Les Editions de Physique Les Ulis, Paris, (1994) Vol. 2A, p. 649.

(33) T. Okuyama, S. Matsumura, N. Kuwano, K. Oki and Y. Tomokiyo: Ultramicroscopy, 31 (1989), 309.

(34) Y. Tomokiyo, T. Okuyama, S. Matsumura, N. Kuwano and K. Oki: Mater. Trans., JIM, 31 (1990), 641.

(35) Y. Tomokiyo, S. Matsumura, T. Okuyama, T. Yasunaga, N. Kuwano and K. Oki; Ultramicroscopy, 54 (1994), 276.

(36) Y. P. Lin and D. M. Bird: Ultramicroscopy, 29 (1989), 233.

(37) Y. Tomokiyo, S. Matsumura, N. Kuwano, M. Kominami, T. Okuyama and K. Oki: J. Electron Microsc., 35 (1986), 359.

(38) T. Okuyama, M. Nakayama, S. Sadamitsu, J. Nakashima and Y. Tomokiyo: Jpn. J. Appl. Phys., 36 (1997), 3359.
(39) J. C. H. Spence and J. Taftø: J. Microsc., 130 (1982), 147.

(40) S. Matsumura, T. Furuse and K. Oki: Diffusion in Ordered Alloys, Ed. by B. Fultz, R. W. Cahn and D. Gupta, TMS, Warrendale, PA, (1993), p. 137.

(41) S. Matsumura, T. Furuse and K. Oki: Mater. Trans., JIM, 39 (1998), 159.

(42) T. Morimura, S. Matsumura, M. Hasaka and T. Tsukamoto: Philos. Mag. A, 76 (1997), 1235.

(43) N. Kuwano, S. Matsumura, T. Furuse and K. Oki: J. Electron Microsc., 45 (1996), 93.

(44) S. Matsumura, J. Hayata and C. Kinoshita: Ann. Rep. HVEM Lab. Kyushu Univ., 20 (1996), 9.

(45) L. Hwang, A. Heuer and T. E. Mitchell: Philos. Mag., 23 (1973), 241.

(46) M. Tokonami and H. Horiuchi: Acta Cryst., A36 (1980), 122.

(47) T. Soeda, S. Matsumura and C. Kinoshita: submitted to Proc. 14th Int'l. Cong. Electron Microscopy, (1998), submitted.

(48) Y. Tomokiyo, T. Kuroiwa and Y. Hayashi: Mater. Trans., JIM, 36 (1995), 1344.

(49) Z. Akase and Y. Tomokiyo: Ann. Rep. HVEM Lab. Kyushu Univ., 20 (1996), 87, and Proc. Asian Science Seminar on New Direction in Transmission Electron Microscopy and Nan-characterization of Materials, Kyushu University Press, Fukuoka, (1998), in press.

(50) F. Izumi, H. Asano, T. Isigaki, E. Takayama-mulomati, Y. Utida, N. Watanabe and T. Nisikawa: Jpn. J. Appl. Phys., 26 (1987), 649. 